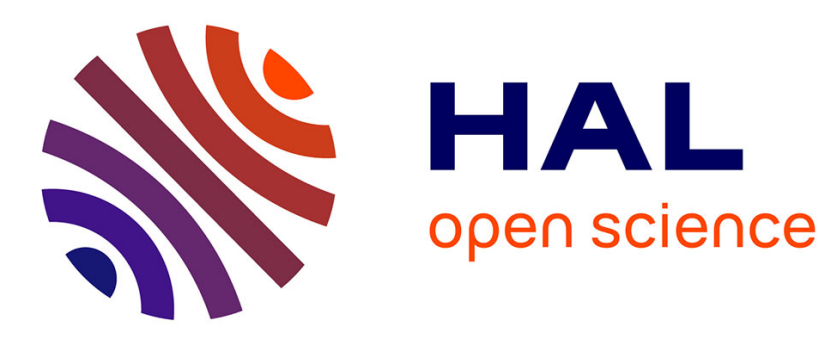

\title{
Evaluation of Multi-Agent Systems: The case of Interaction
}

\author{
Hussein Joumaa, Yves Demazeau, Jean-Marc Vincent
}

\section{To cite this version:}

Hussein Joumaa, Yves Demazeau, Jean-Marc Vincent. Evaluation of Multi-Agent Systems: The case of Interaction. Proceedings of the 3rd International Conference on Information \& Communication Technologies: from Theory to Applications (ICTTA'08), 2008, Damascus, Syria. hal-00953626

\section{HAL Id: hal-00953626 \\ https://hal.inria.fr/hal-00953626}

Submitted on 28 Feb 2014

HAL is a multi-disciplinary open access archive for the deposit and dissemination of scientific research documents, whether they are published or not. The documents may come from teaching and research institutions in France or abroad, or from public or private research centers.
L'archive ouverte pluridisciplinaire HAL, est destinée au dépôt et à la diffusion de documents scientifiques de niveau recherche, publiés ou non, émanant des établissements d'enseignement et de recherche français ou étrangers, des laboratoires publics ou privés. 


\title{
Evaluation of Multi-Agent Systems: The case of Interaction
}

\author{
Hussein Joumaa, Yves Demazeau, Jean-Marc Vincent \\ LIG - IMAG \\ 46, Avenue Félix Viallet, 38031 \\ Grenoble CEDEX1 - France \\ Email: \{Hussein.Joumaa, Yves.Demazeau, Jean-Marc.Vincent\}@imag.fr \\ Telephone: +334765747 76 \\ Fax: +33476574602
}

\begin{abstract}
This paper deals with the evaluation of Multi-Agent Systems (MAS) at the level of their interaction. Two problems that may be a bias in the evaluation and measurement of interaction are discussed. The first one is the difference between the quantities of information carried by a unit of interaction in two systems having different architectures. The second one concerns the interaction units that are received and cannot be exploited by the agent. In this work, an evaluation based on the weight of the information brought by an interaction is suggested. In order to achieve this, a MAS model, on which the evaluation is based, is defined. Then, the different problems and solutions which will help to evaluate the interaction are studied. Finally, the approach is applied on two different implementations that solve the same problem.
\end{abstract}

Index Terms-Multi-Agent Systems, Evaluation, Multi-Agent System model, Interaction.

\section{INTRODUCTION}

Multi-Agent Systems have become increasingly important in computer science and their application domains are growing in number and complexity. The strength of this paradigm comes from the flexibility and variety of interaction types and organization models present in such systems.

However, the distributed nature of MAS and the complexity of the interaction inside them make their understanding and analysis a hard task. Due to this complexity, it is interesting therefore to redeem some fundamental aspects which characterize the MAS. [1] defines a MAS as a set of organized agents acting in a common environment. This statement puts a stress on two dimensions: interactions and organization. The present work consists in considering the interaction, the most important characteristic of complex software according to [2], as a problematic of evaluation, therefore allowing study and comparison of MAS at the level of their interactions.

The evaluation of MAS interests in the computation of the parameters making possible to understand the behaviour, and to compare the various systems between them(section 2).

Such an evaluation of interactions brings up different types of problems:

- The effect of an interaction unit (the message, according to the proposed model (section 3)) in a system may be equivalent to "n" units in another system (problem 1)
- The interaction units that are received and cannot be used by the agent may be a bias in the measurement of interaction in MAS (problem 2)

As such, an evaluation at the interaction level cannot be done without dealing with these problems from the point of view of the quantity of information carried by messages and the way they are used. In this work, an evaluation based on the weight of the information brought by a message is suggested. Consequently, an approach to relate the notion of message weight to measurable notions is proposed.

In order to achieve this, a MAS Model, on which the evaluation is based, is defined in section 3 .

In section 4, different problems and solutions are studied to evaluate the interaction. First of all, to deal with problem 2 , the notion of a pertinent interaction (pertinent message) is defined, according to which a pertinent message is a message that changes the internal state of an agent or starts off an action. Therefore, the solution to problem 2 is to focus on the measurement of the quantity of pertinent information rather than the total quantity of information.

Regarding problem 1, a function $\Phi$ is defined in order to calculate the weight of pertinent messages (a null weight is given to other messages). The relation between the received message $m$ and the effects on the agent is studied in order to calculate the $\Phi(m)$ value. According to the model, two kinds of functions are considered:

- A function that associates weight to the message according to its type.

- A function that associates weight to the message according to the change provoked on the internal state and the actions triggered by its reception.

The approach then focuses on the evaluation of interactions in MAS based on this function. Finally, to illustrate this approach, it is applied on two different implementations which solve the same problem. This application is constituted of two principal sets of agents (transporters and explorers). The agents have to explore a space, which is in principle unknown, in order to find, recover and transport the discovered ore until the base. 


\section{STATE OF THE ART}

Several works consider the agent's interaction as an evaluation criterion but none of these aims at evaluating the interaction in MAS. In this part, some works on the evaluation of MAS, basically those which consider the interaction as criterion, will be presented. There are four types of works related to the evaluation in MAS:

- Evaluation of Agent Oriented Software Engineering (AOSE) Methods: this part concerns works on comparing agent-oriented methodologies based on the software engineering related criteria and characteristics of MAS. Mylopoulos in [3] tries to evaluate the structures of organization for TROPOS methodology [4]. Ten structures of organization and nine criteria have been proposed. The various organizations are evaluated according to these criteria. Mylopoulos proposed giving for each criterion a value among: sufficiently negative (-), negative () , positive $(+)$, and sufficiently positive $(++)$. In this work, a certain number of criteria are evaluated based on the agent's interaction such as modularity, cooperativity, adaptability, predictability. Cernuzzi and Rossi compare in [5] two AOSE methodologies: the agent modeling techniques for systems of BDI Agent and MASCommonKADS. They propose quantitative evaluation attributes concerning the characteristics of the agent and those related to the interaction process. In [6], O'Malley and Deloach propose a number of criteria, including the interaction criterion, for evaluating methodologies to allow choosing between AOSE methodologies and Object Oriented (OO) methodologies.

- Evaluation of MAS Architecture: Davidsson and Johanson [7] propose evaluating the MAS Architectures in terms of three-dimensional space: the determination of the set of possible applications, the determination of the set of possible architectures, and the definition of the set of attributes used to evaluate the architecture. They consider communication (according to the proposed model(section 3),communication is equivalent to interaction) as a characteristic of the evaluation.

- Evaluation of MAS: Bincheng et al. [8] study the relationships between a local agent's behavior and global MAS performances. The interaction is considered as a basic characteristic at the local behavior and as a characteristic of measurement for global MAS performance. Joumaa in [9] tries to find the criteria which will be used for the comparison of MAS between them. Then, the openness and the adaptability of MAS are studied. The quantification of these characteristics is based on the measurement of the interaction.

- Evaluation of Multi-Agent Platforms: Jurasovic et al. [10] compare two versions of MAS, designed using Grasshopper agent platform while the other was designed using Jade, at the level of their interactions. The two versions are almost identical in terms of their software architecture.
The interaction is considered as a basic criterion of evaluation. More complex attributes and characteristics are evaluated based on its measurement. The problem with all the presented classic evaluations is that they consider in the measurement the quantity of interactions but not the weight of the carried information. The actual work tries to resolve this problem on a general MAS Model presented in the following section.

\section{MAS MODEL}

In this section, a MAS model, on which the evaluation is based, will be defined. Firstly, the notions of agent and environment will be introduced. Then, the process occurring in a MAS will be discussed and formalized.

\section{A. Agent and Environment}

An agent is a physical or virtual entity evolving in an environment. It has only a partial representation of this environment. It is able to perceive and to act upon it, and to interact with other agents $[11,12]$. According to $[2,13]$, an agent encapsulates an internal state (non accessible to other agents), and makes decision about what to do based on this state, without the direct intervention of other entities.

Based on these definitions, three different dimensions can be distinguished in the modeling of the architecture of an agent: interaction, decision making, and the internal state. As Figure 1 shows, the agent is modeled as a system composed of: a processing unit which is the part of the agent which deals with decision dimension. The decisions are made, based on the internal state of the agent. A memory represents the internal state of the agent. An agent has the capability to act and interact by means of an interface used for reception, and also an interface used to send messages. The messages received are stocked in a stack of received messages. The function of this stack is to register the messages received when realizing the processing of another message.

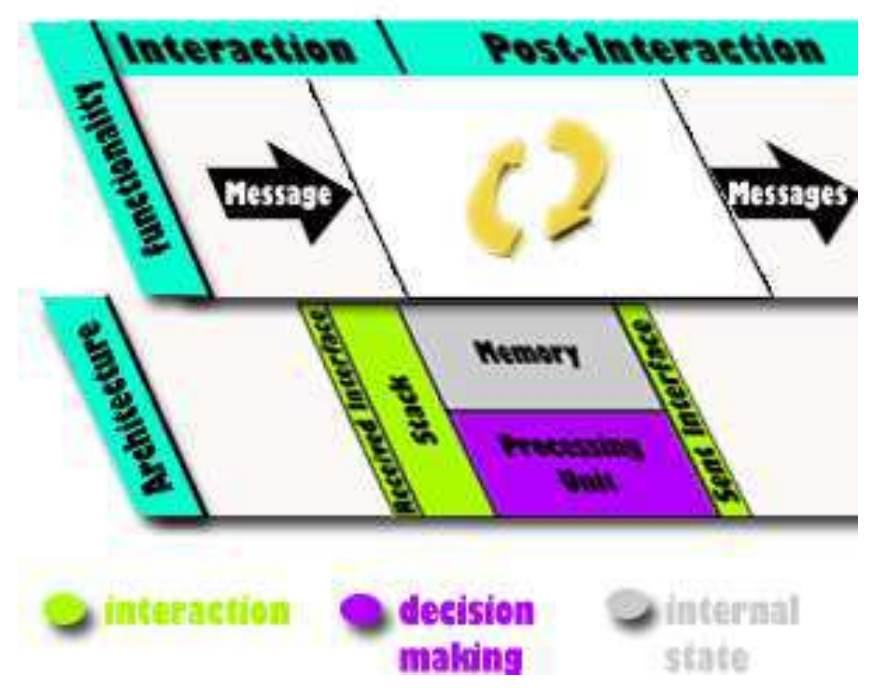

Fig. 1. Architecture and functionality of an agent 
In this work, the messages are the only way to interact between the agent and the other entities in the MAS (the other agents and the environment). An agent comprises three successive phases: message reception, processing and action. The action of the agent is translated by sending one or more messages to the other entities. An agent may take certain initiatives. Therefore, it may send messages without being stimulated.

The environment is the common medium shared by the set of agents. The environment has the following properties:

- Accessible: an agent can, with its own capabilities, perceive the state of the environment.

- Determinist: the future state of the environment is function of its current state and the actions of the agent.

- Static: the state of the environment is stable (does not change) without the act of an agent.

- Discrete: The number of actions able to be made upon the environment and the states of it are finite.

In this model, the environment is considered as a passive entity which has the same architectural dimensions as an agent. It reacts to the actions of agents on it, and responds to their requests. The difference between the agents and the environment is at the level of their functionality but not at the level of their architecture. This architecture guarantees the properties of the environment cited above.

\section{B. Interaction and Post-interaction}

A MAS is a set of agents which evolve in a common environment. In this model, the process occurring inside a MAS is divided into two main steps: Interaction and PostInteraction.

Interaction is represented by sending and receiving messages between the two entities "Sender" and "Receiver". On one hand, from the point of view of the "Sender" entity, the interaction is performed by sending one message among the set of messages that is able to send. On the other hand, from the point of view of the "Receiver" entity, the interaction consists in the reception of a message among the messages that it understands or a message that appears incomprehensible. Those "Sender" and "Receiver" entities could be either agents or environment. The following notations are used:

- $M_{\text {Sent }}^{A}$ : The set of messages which may be sent by the agent $A$ and $m_{\text {Sent }, i}^{A}$ is the message sent by the agent $A$ at the instant $i$.

- $M_{\text {Received }}^{A}$ : The set of messages which may be received by the agent $A$ and $m_{\text {Received,i }}^{A}$ the message received by the agent $A$ at the instant $i$.

- $m_{\phi}^{A}$ designates a message unable to be understood by the agent $A$.

Therefore:

$$
\text { Interaction }: M_{\text {Sent }}^{A} \rightarrow M_{\text {Received }}^{B} \cup\left\{m_{\phi}^{B}\right\}
$$

The process of interaction is a function that associates for a sent message $m_{\text {Sent }, i}^{A}$ a received message $m_{\text {Received }, j}^{B}$ or an incomprehensible message $m_{\phi}^{B}$ and $j \succ i$.
In the following, the step of post-interaction is discussed. At the agent level, the post-interaction consists in the processing of the message and then the action. The processing is realized by: memorization that treats the part of change at the internal state caused by the received message, and the decision that concern the choice of the action that will be handled. The following notations are used:

- $S^{A}$ : The set of possible internal states for the agent $A$ and $s_{i}^{A}$ is the internal state of the agent $A$ at the instant $i$.

- $A C^{A}$ : The set of actions which may be done by the agent $A$ and $a c_{i}^{A}$ is the action done by the agent $A$ at the instant $i$. $a c_{\phi}^{A}$ designates that no action triggered by the agent $A$.

The treatment of a message by an agent is done therefore in two steps:

- The memorization step is realized by the function $M E M^{A}$. This function associates a new internal state to a received message and the internal state current.

$$
M E M^{A}: M_{\text {Received }}^{A} \times S^{A} \rightarrow S^{A}
$$

- The decision step is realized by the function $D E C^{A}$ This function associates an action to be executed to the current internal state and the received message.

$$
D E C^{A}: M_{\text {Received }}^{A} \times S^{A} \rightarrow A C^{A} \cup\left\{a c_{\phi}^{A}\right\}
$$

In the following section, the evaluation of the interaction is studied based on this model.

\section{STudy Of THE CASE Of INTERACTION}

In this section, different problems, which arise in the evaluation of the interaction, are studied. First of all, the different aspects and levels of abstractions, according to which such an evaluation may be realized, will be discussed. Then, the problems that encounter this evaluation and the proposed solutions will be listed.

\section{A. Levels and aspects of evaluation}

At the beginning of the evaluation, the aspects and levels of abstraction, according to which the interaction will be evaluated, must be specified. The interaction in MAS can be evaluated according to two different levels of abstraction:

- Micro level: the evaluation at micro level involves quantitative and qualitative analysis within and between the entities. In this case, the study of the interaction is done at the agent's level.

- Macro level: the study at this level is conducted on the system as a whole. In a MAS, the analyses are done on the set of organized agents and the environment.

In this work, an evaluation at the micro level (agent's level) is realized. Then, the problem of interaction evaluation, at the MAS level, is studied. Therefore, according to [11] the function in a MAS is considered as the sum of the function at the agent's level with the collective function :

$$
\text { Function }(M A S)=\sum \text { Function }(\text { Agent })+\gamma
$$


$\gamma$ designates the collective function.

In this evaluation, the function of interaction is only studied. As the model shows, the interaction is considered as having effects on one agent. There is not a collective function at this basic level. The collective function $\gamma$ in this case adheres to more complicated properties (coordination, co-operation) which exceed the limits of this work. Then, the value of $\gamma$ is null. The interaction at the level of MAS is considered as the sum of the interactions that occurs at the agent's level.

The interaction can be evaluated according to the following aspects:

- The structural aspect refers to the topology, the structure and the properties of the interaction network between the agents.

- The statistical aspect is the quantification of usual magnitudes such as the number of messages and their size.

- The the syntactical refers to the typology of messages and the complexity of their contents.

The study of the structural aspect is not linked to the choices made for evaluating the interaction at the "micro level". The study of the interaction network concerns the set of agents (the MAS) but not the single agent. However, the statistical and syntactical aspects are studied in this paper. These aspects are considered to ensure the evaluation of the interaction that concerns the weight of interactions between agents.

\section{B. Problems of evaluating interaction}

As shown in section 2, in a classic evaluation, all the interactions are considered having the same weight in the measurement. Then, such an evaluation of interaction brings up different types of problems:

- The effect of a message in a system may be equivalent to "n" units in another system. This problem 1 is modeled as follows:

For the reception of message $m$ (the actual internal state is $s_{k}^{A}$ ) by agent $A$,

$$
D E C^{A}\left(m, s_{k}^{A}\right)=a c_{k+1}^{A}, M E M^{A}\left(m, s_{k}^{A}\right)=s_{k+1}^{A}
$$

For the reception of a set of messages $\left\{m_{i}\right\}_{i=l, \ldots, l+n}$ by the agent $B$ (the actual internal state is $s_{l}^{B}$ and $s_{k}^{A} \Leftrightarrow s_{l}^{B}$ ):

$$
D E C^{B}\left(m_{i}, s_{i}^{B}\right)=a c_{i+1}^{B}, M E M^{B}\left(m_{i}, s_{i}^{B}\right)=s_{i+1}^{B}
$$

with: $\bigcup a c_{i}^{B} \Leftrightarrow a c^{A}$ and $s_{l+n+1}^{B} \Leftrightarrow s_{k+1}^{A}$.

In this case, the same results need more interactions. This may be a bias to the study and the comparison of the interactions in these systems. In the first system, the weight of the interactions realizing the same work is " 1 " and it is " $n$ " in the second. (problem 1)

- The problem 2 is the interaction units that are received and cannot be used. This problem is expressed as follows: For the reception of message $m$ by agent $A$ :

$$
D E C^{A}\left(m, s_{i}^{A}\right)=a c_{\phi}^{A}, M E M^{A}\left(m_{i}, s_{i}^{A}\right)=s_{i+1}^{A}
$$

with: $s_{i+1}^{A} \Leftrightarrow s_{i}^{A}$. These messages, which are considered in the measurement of the interaction, may be also bias in the evaluation of interaction in MAS. (problem 2)

\section{Evaluating pertinent interactions}

In the following, the notion of pertinent interaction (pertinent message) is defined. A pertinent message is a message that changes the internal state of an agent or starts off an action. Then, $m$ (received by the agent $A$ ) is a pertinent message if:

$$
D E C^{A}\left(m, s_{i}^{A}\right)=a c_{i+1}^{A}, M E M^{A}\left(m, s_{i}^{A}\right)=s_{i+1}^{A}
$$

with: $s_{i+1}^{A} \leftrightarrow s_{i}^{A}$ or $a c_{i+1}^{A} \neq a c_{\phi}^{A}$.

Therefore, the proposed solution to problem 2 is to focus on the measurement of the quantity of pertinent information rather than the total quantity of information.

Regarding problem 1 , a function $\Phi$ is defined to calculate the weight of pertinent messages (A null weight is given to other messages). Therefore, the decision of the agent is made on the received message, the first idea is to divide the set of possible received messages into sub-sets having the same type. Then, a weight is associated to a message according to its type. The distinction between the types of messages depends on the application of the MAS, which is to be evaluated. In the literature there are works that adopted the primitives proposed by [14] to the type of interaction. This work consists of four possibilities of message types: present, request, answer, and inform. These four types have to be distinguished because of the different basic behaviors that they model from the sender or the receiver points of view. A "request" includes a change of state of the sender, waiting for the answer. An "inform" includes no change of state for both the sender and the receiver. It might generate other informs, and possibly answers. A "present" includes a possible change in the state of the sender and/or of the receiver. Typically, a "present" will enable entering a society and introduce itself to other agents. This solution partially resolves the problem and it works when two messages of the same type have equivalent effects on the agent.

Another proposed solution is to associate the weight of the message to the results of its treatment. As the proposed model, the treatment of a message is divided into two functions: Decision and Memorization. This solution proposes dividing the function $\Phi$ into two functions $\Phi_{D E C}$ and $\Phi_{M E M}$ :

- The function $\Phi_{M E M}$ associates a value to the variation of the internal state (caused by memorization step). To quantify, some measurable characteristics of the internal state must be defined. The specification of these characteristics is related to the application. The variation on one of these characteristics having a weight, then the function $\Phi_{M E M}$ is considered as the sum of these weights.

- Concerning $\Phi_{D E C}$, this function associates a value to the triggered actions (results of decision step). To quantify, certain type of actions must be defined. A type of actions having a weight. Then, the value of the function $\Phi_{D E C}$ is considered as the sum of the weights of triggered actions.

Then, the function $\Phi$ is defined as the sum of these functions. The specification of the characteristics of the internal state, 
their weights and the types of actions and their weights is the task of the evaluator.

In the following section, this approach is applied on two different implementations that solve the same problem.

\section{EXPERIMENTATION}

The application is the simulation of societies of robots having the goal to collect pieces of ores [15]. Agents have a vision range, perceive all other agents within their vision range, and communicate one-to-one by messages. The agents move in the eight directions. Two agents cannot locate in the same time at the same place. They consume energy when acting. The agents die when their batteries energy is consumed. There are three types of agents: bases, explorers and transporters.

The base basic behavior is to listen messages, receive information, and decide the general course of action. The agents can acquire an additional energy at the base. The transporters give the system the competence to pick up ore. They collect the ores detected by the explorers. An explorer is the agent that displays the most autonomous behavior. The explorer basic behavior is to go to a new position in the world and prospects it for ore. It communicates with the friendly units within its communication range and sends information about the ores. Moreover, a number of additional rules and constraints (specifications) are added mainly to help in the analysis of the problem and the design of the solution system. The experimentation is done on two MAS implemented with different specifications. In the following, the principal difference between the 2 systems (especially at the interaction level) is showed.

In MAS1, the explorers should make the initiative to communicate (acting first). When an explorer found ore. It sends a message "found an ore". The transporters process the broadcast and communicates directly back to the explorer if it wants to pick the ore up. The explorer evaluates all transporters and decides which transporter is best for the job. It then sends a message directly to the transporter. It receives the message and sends an "OK" to the explorer. When the explorer receives the ok it moves to find the next ore.

In MAS2, the explorer asks the base in which area to look for ore. When it has reached the area it perceive the environment for ore and afterward moves onto the ore positions to "verify" the position, after this it's able to communicate its knowledge about ore positions with transporter. When an explorer is done surveying the area is returns to the base and ask for a new area. When the energy is low the explorer returns to a base for a recharge. The transporter has the job of collecting ore located by the explorer. Transporter will contact explorers to communicate about which ore positions to check. When an ore position is reached the transporter probe the environment to check if the ore (still) is in place, and if so picks it up. As the transporter only can carry one ore, it returns to a base and unload the ore (telling the base the position where it was picked up), after a recharge it continues collecting ore. The base contains the ore collected and has knowledge about where it was picked up (obtained by communicating with transporter unloading ore). This information is used to decide where to send an explorer asking for a position/ area to explore. The base also creates an internal map, divided into zones, of the surrounding planet, which in combination with the information about collected ore is used when guiding the explorers. When the base has reached its goal it sends a message to all robots to return to the base, ready for take of.

In the following, the results of experimentation are showed. The measurement on the MAS is realized with the same parameters of the experimentation and in the same hardware environment. First of all, the Figure2 shows the variation of sent message numbers with number of collected ore. To collect 80 pieces of ores the number of messages sent in MAS1 is 10800 and 1900 in MAS2. The large number of messages sent in MAS1 is due to the protocol of interaction described above. The Figure1 presents the quantity of information transmitted in these systems but not the weight of the information transmitted. In the following of the section, the approaches concerning the weight of the message received will be applied.

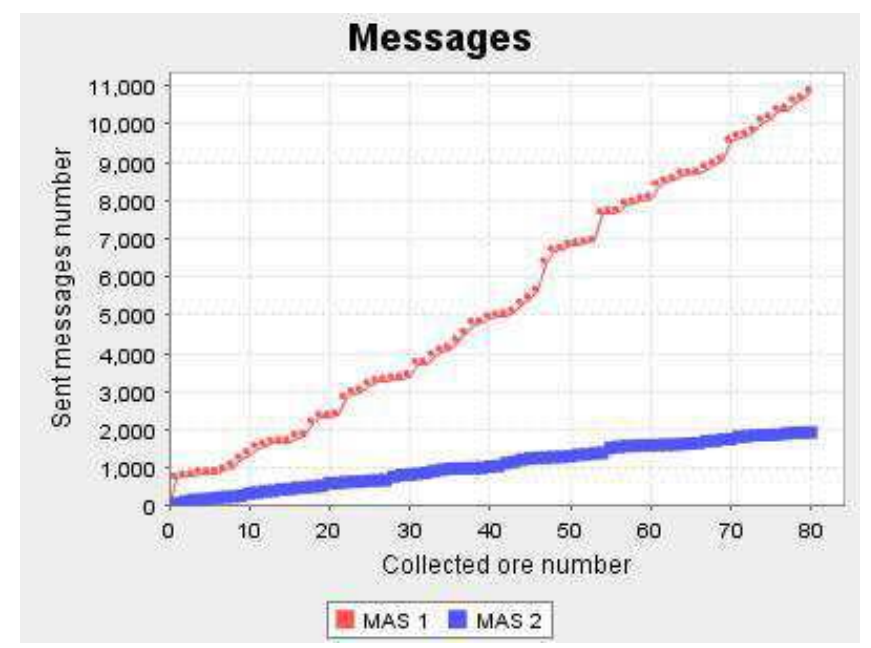

Fig. 2. The variation of send message with number of collected ore

To apply the type approach described in section 4 , the types of messages must be distinguished. The distinction between these types depends on the application as discussed above. For example, For the MAS1 the types of messages received by an explorer agent are: messages received during the communication with transporters, the message "OK" received when the mission is allowed to a transporter, refuel messages received from the base, the message of returns received from the base, and messages received from the environment. A value among "high" (1), "medium" (0.75), "low" (0.5), and "non pertinent message" (0) is affected for each type of messages. This value is affected depending on the importance of the message for the agent. The results of experimentation are showed in Figure3. In this approach, the sent message weight to collect 80 pieces of ore for MAS1 is 4700 and 1400 for MAS2. This approach considers that the weight of all the messages having the same type is equivalent. 


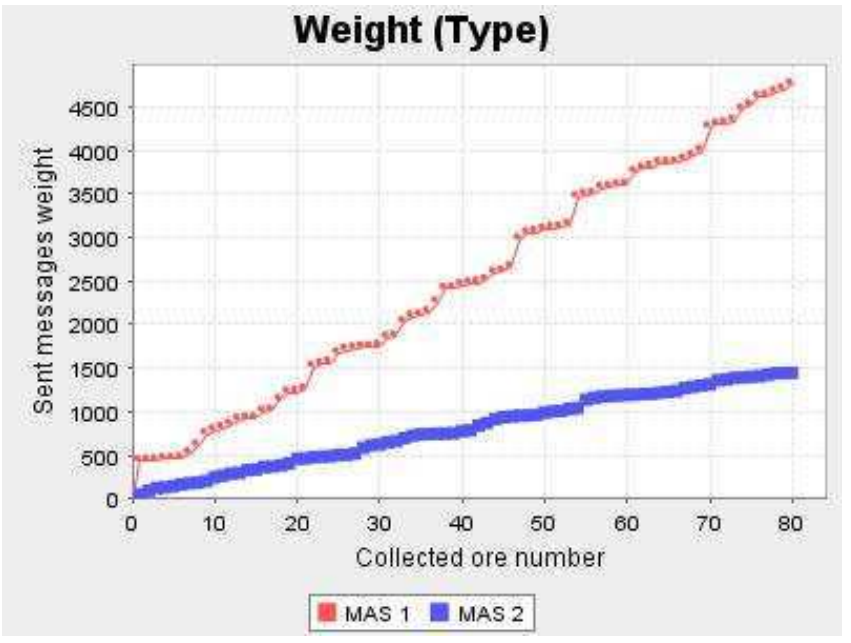

Fig. 3. The variation of messages Weight (type) with number of collected ore

In the following, the approach that associates the weight of the message to the results of its treatment is applied. As mentioned in section 4 , some measurable characteristics of the internal state are defined (For example, for the base in MAS1 these characteristics are: the information stocked about quantity of ore detected, quantity of fuel, and ores position). A weight between 0 and 0.5 is associated for the variation on each one of these characteristics. Then, the function $\Phi_{1}$ is considered as the sum of these weights. Concerning $\Phi_{2}$, the types of actions may be triggered by agents is defined. A weight between 0 and 0.5 is associated to each action. The weight of the received message is the sum of $\Phi_{1}$ and $\Phi_{2}$ as proposed in section4. The results of experimentation are showed in the Figure4. In this approach the sent message weight to collect 80 pieces of ore for MAS1 is 2800 and 1200 for MAS2.

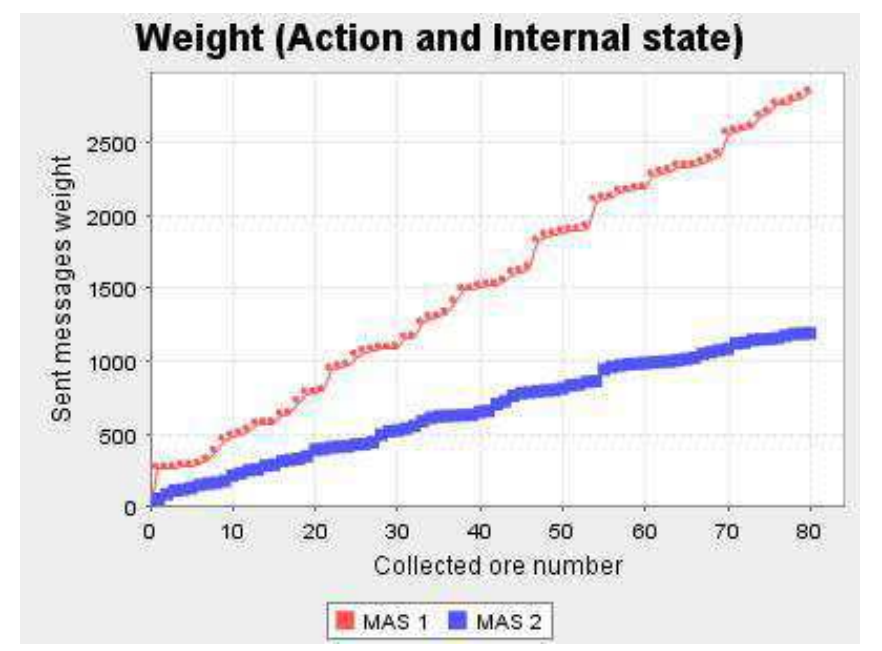

Fig. 4. The variation of messages Weight (action and internal state) with number of collected ore

\section{CONCLUSION}

In this paper, the evaluation of Multi-Agent Systems (MAS), based on the proposed model, at the level of their interaction has been discussed. An approach based on the weight of the information brought by an interaction has been proposed to resolve the problems being a bias in the evaluation. Then, the approach has been experienced on two different implementations that solve the same problem. It is important to notice that this study is the base of another works on the evaluation of the cooperation and the coordination in a MAS.

\section{REFERENCES}

[1] Y. Demazeau, From interactions to collective behaviour in agent-based systems, First European conference on cognitive science, 1995.

[2] M. Wooldridge and P. Ciancarini, Agent Oriented Software Engineering. the state of the art, First international Workshop on Agent-Oriented Software Engineering, 2000.

[3] J. Mylopoulos, M. Kolp, and P. Giorgini, Agent-Oriented Software Development, SETN, 2002.

[4] P. Bresciani, P. Giorgini, F. Giunchiglia, J. Mylopoulos, and A. Perini, Tropos: An Agent-Oriented Software Development Methodology, Journal of Autonomous Agents and Multi-Agent Systems, 2004.

[5] L. Cernuzzi and G. Rossi, On the evaluation of agent oriented modeling methods, Agent Oriented Methodology Workshop, 2002.

[6] S. OMalley and S. DeLoach, Determining when to use an agent-oriented software engineering methodology, Second International Workshop On Agent- Oriented Software Engineering, 2001.

[7] P. Davidsson and S. Johansson, Evaluating Multi-Agent System Architectures: A case study concerning dynamic resource allocation, Third International Workshop on Engineering Societies in the Agents, 2002.

[8] H. Bincheng, L. Jiming, and J. Xiaolong, From local behaviors to global performance in a Multi-Agent System, Intelligent Agent Technology, 2004.

[9] H. Joumaa, Y. Demazeau, and J. M. Vincent, Method for quantitative evaluation of Multi-Agent System, unpublished.

[10] K. Jurasovic, G. Jezic, and M. Kusek, A performance Analysis of Multi-Agent Systems, International Transactions on Systems Science and Applications, 2006.

[11] Y. Demazeau and A. R. Costa, Populations and organisations in open multi-agent systems, First Symposium on parallel and Distributed Artificial Intelligence, 1996.

[12] J. Ferber, Les Systemes Multi-Agents: vers une intelligence collective, InterEditions, 1995.

[13] M. Wooldridge and N. Jennings, Intelligent agents: Theory and practice, The Knowledge Engineering Review, 1995.

[14] G. Gaspar, Communication and Belief Changes in a Society of Agents Towards a Formal Model of Autonomous Agent, D.A.I. 2, 1991.

[15] Y. Demazeau, Examination 2004, University South Denmark - Odense, 2004. 